\title{
Search for Dark Particles and Dark Sector at Belle
}

\author{
Eunil Won*t \\ Korea University \\ E-mail: eunilwon@korea.ac.kr \\ for the Belle Collaboration
}

\begin{abstract}
We search for dark photon $A^{\prime}$ and the dark Higgs boson $h^{\prime}$ particles that are suggested in number of recently proposed dark sector models. We present our search results in the production of $e^{+} e^{-} \rightarrow A^{\prime} h^{\prime}$ with the decay mod of $h^{\prime} \rightarrow A^{\prime} A^{\prime}$. The search was carried out in both inclusive and exclusive modes. We also discuss a search for new dark vector gauge boson that couples to light quark predominantly.
\end{abstract}

ICHEP 2018, International Conference on High Energy Physics

4-11 July 2018

Seoul, Republic of Korea

\footnotetext{
* Speaker.

${ }^{\dagger}$ For the Belle collaboration.
} 


\section{Introduction}

The dark particles, can be a candidate for dark matter were originally considered as a new spin1 boson for new physics [1] beyond the Standard Model [2, 3]. The dark boson $A^{\prime}$ can be produced in a radiative $e^{+} e^{-}$collision via kinetic mixing of a virtual photon with the kinetic mixing parameter $\varepsilon$ and is illustrated in the left diagram of Fig. 1. This new dark boson requires an extended Higgs sector to break the new $U(1)^{\prime}$ symmetry and is commonly referred as the dark Higgs $h^{\prime}$. In this presentation, we search for the dark boson and the dark Higgs in both exclusive and inclusive modes.

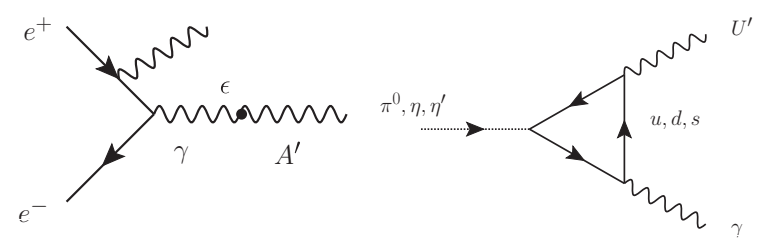

Figure 1: Example Feynman diagrams that illustrate production of the dark photon $A^{\prime}$ in $e^{+} e^{-}$collision and of the dark boson $U^{\prime}$ from light mesons.

Also, recently there is a proposal of a new dark gauge boson that couples predominantly to light quarks [4], its production example is shown in Fig. 1, and we also discuss a search for the new dark gauge boson $U^{\prime}$.

\section{Experimental facility and the data set}

We use data collected with the Belle detector [5] at the KEKB $e^{+} e$ collider [6] , amounting to $977 \mathrm{fb}^{-1}$ at center-of-mass energies corresponding to the $\Upsilon(1 S)$ to $\Upsilon(5 S)$ resonances and in the nearby continuum. We optimize the selection criteria and determine the $e^{+} e \rightarrow A^{\prime} h^{\prime}$ signal detection efficiency using a Monte Carlo (MC) simulation where the interaction kinematics and detector response are simulated with the packages MadGraph [7] and GEANT3 [8], respectively.

\subsection{Search for dark photon analysis}

We study the Higgs-strahlung channel, $e^{+} e^{-} \rightarrow A^{\prime} h^{\prime}$. The dark photon $A^{\prime}$ can decay into lepton pairs, hadrons, or invisible particles while the dark Higgs boson $h^{\prime}$ can decay into either $A^{\prime}, A^{\prime(*)}$, leptons pairs, or hadrons, where $A^{\prime *}$ is a virtual dark photon [9]. In total there are 10 exclusive channels: $\left.3\left(\ell^{+} \ell^{-}\right), 2\left(\ell^{+} \ell^{-}\right)\left(\pi^{+} \pi^{-}\right),\left(\ell^{+} \ell^{-}\right) 2 \pi^{+} \pi^{-}\right)$, and $3\left(\pi^{+} \pi^{-}\right)$. We also look at 3 inclusive modes for $m_{A^{\prime}}>1.1 \mathrm{GeV} / c^{2}: 2\left(\ell^{+} \ell^{-}\right) X$ where $X$ is the missing mass from the dark photon candidate.

For the background estimation, we use the same esign events from $e^{+} e^{-} \rightarrow\left(\ell^{+} \ell^{+}\right)\left(\ell^{-} \ell^{-}\right)$ $\left(\ell^{+} \ell^{-}\right)$, order masses of lepton (or hadron) pairs as $m_{\ell \ell}^{1}>m_{\ell \ell}^{2}>m_{\ell \ell}^{3}$, and plot $m_{\ell \ell}^{1}-m_{\ell \ell}^{3}$ vs. $m_{\ell \ell}^{1}$. The background estimation from MC events and real data agree each other and no significant access of signal events is seen in the data.

The upper limit on the $\alpha_{D} \times \varepsilon^{2}$ are computed based on equations described in Ref [9]. Figure 2 shows the $90 \%$ credibility level (CL) upper limits on $\alpha_{D} \times \varepsilon^{2}$ for Belle, expected and measured, 
and for BaBar, for five different mass hypotheses for the dark Higs boson (top row) and dark photon (bottom row) masses.
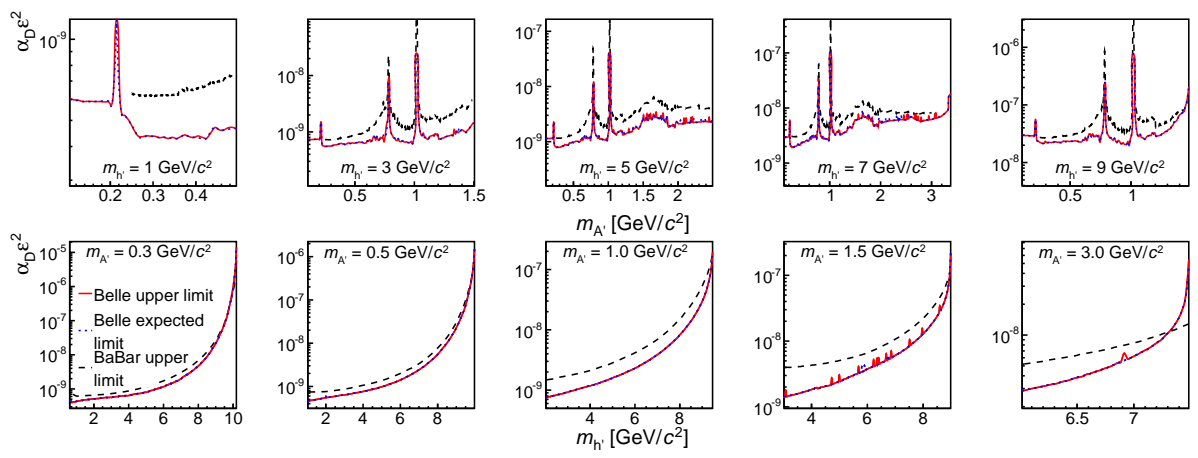

Figure 2: $90 \%$ CL level on the product $\alpha_{D} \times \varepsilon^{2}$ versus dark photon mass (top row) and dark Higgs boson mass (bottom row) for Belle (solid red curve) and BaBar [11] (dashed black curve). The blue dotted curve, which coincides more or less with the solid red curve, shows the expected Belle limit. The figure is taken from [10].

\subsection{Search for dark boson analysis}

We search for $U^{\prime}$ bosons decaying to $\pi^{+} \pi^{-}$pairs using $\eta \rightarrow \pi^{+} \pi^{-} \gamma$ decays where $\eta$ is produced in the decay chain $D^{*+} \rightarrow D^{0} \pi^{+}, D^{0} \rightarrow K_{S}^{0} \eta$. The decay, $U^{\prime} \rightarrow \pi^{+} \pi^{-}$, is expected to have a relatively small branching ratio down to $10^{-2}$ [4] but nevertheless provides a very clean environment to look for a possible dark vector gauge boson.

After reconstructing all needed final-state particles, we define the $\eta$ signal region as $M\left(\pi^{+} \pi^{-} \gamma\right)$ $\in[535.5,560.5] \mathrm{MeV} / c^{2}$, and the sideband regions used for background subtraction as $M\left(\pi^{+} \pi^{-} \gamma\right) \in$ $[520.0,532.5]$ or $[563.5,576.0] \mathrm{MeV} / c^{2}$. The $M\left(\pi^{+} \pi^{-}\right)$distribution for the background-subtracted $\eta$ signal is shown in Fig. 3.

We set a 95\% confidence level upper limits on $\alpha_{U^{\prime}}$ using the Feldman-Cousins approach [12], adding the statistical and systematic uncertainties in quadrature. The upper limit as a function of the $U^{\prime}$ boson mass is shown in Fig. 3 . Considering other results in this mass region, we find that our limit is stronger than that from a model-dependent analysis [4] of the $\phi \rightarrow e^{+} e^{-} \gamma$ decays [13] for $m_{U^{\prime}}>450 \mathrm{MeV} / c^{2}$, but weaker than the limit based on the $\eta \rightarrow \pi^{0} \gamma \gamma$ total rate [4].

\subsection{Other Ongoing Analyses}

We are also working on a long lived dark photon search where $c \tau$ can be as long as $1-10 \mathrm{~cm}$. Since it is low multiplicity final state (two charged tracks and one photon), the efficiencies are low in general in Belle and one analysis is ongoing. Another analysis is a search for invisible decays when the dark photon decays to a pair of dark matter particles [14]. Since this is a final state with a single photon only, the Belle analysis relies on the photon conversion technique.

\section{References}

[1] P. Fayet, "Effects of the Spin-1 Partner of the Goldstino (Gravitino) on Neutral Current Pheomenology," Phys. Lett. B 95, 285 (1980). 

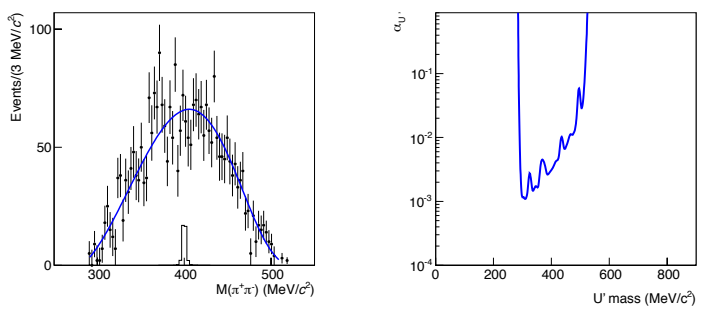

Figure 3: Left: $\pi^{+} \pi^{-}$invariant mass distribution from the $\eta \rightarrow \pi^{+} \pi^{-} \gamma$ signal (points with error bars), the fitted differential decay rate (solid curve), and an example $U^{\prime}$ signal at a mass of $400 \mathrm{MeV} / \mathrm{c}^{2}$ from $\eta \rightarrow U^{\prime} \gamma, U^{\prime} \rightarrow \pi^{+} \pi^{-}$(histogram with arbitrary normalization). Right: Computed $95 \%$ upper limit on the baryonic fine structure constant $\alpha_{U^{\prime}}$ as a function of the unknown $U^{\prime}$ mass (solid curve).

[2] G. Aad et al., [ATLAS Collaboration], "Summary of the ATLAS experiment's sensitivity to supersymmetry after LHC Run 1 - interpreted in the phenomenological MSSM," J. High Energy Phys. 10, 134 (2015).

[3] S. Chatrchyan et al., [CMS Collaboration], "Interpretation of searches for supersymmetry with simplified models,” Phys. Rev. D 88, 052016 (2013).

[4] S. Tulin, "New weakly coupled forces hidden in low-energy QCD", Phys. Rev. D 89, 114008 (2014).

[5] A. Abashian et al. [Belle Collaboration], Nucl. Instrum. Methods Phys. Res. Sect. A 479, 117 (2002); also see detector section in J.Brodzicka et al., Prog. Theor. Exp. Phys. 2012, 04D001 (2012).

[6] S. Kurokawa and E. Kikutani, Nucl. Instrum. Methods Phys. Res. Sect. A 499, 1 (2003), and other papers included in this Volume; T. Abe et al., Prog. Theor. Exp. Phys. 2013, 03 A001 (2013) and following articles up to 03A011.

[7] J. Alwall P et al., "The automated computation of tree-level and next-to-leading order differential cross sections, and their matching to parton shower simulations", J. High Energy Phys. 07, 079 (2014).

[8] R. Brun et al., GEANT 3.21, Cern/DD/ee/84-1, (1986).

[9] B. Batell, M. Pospelov, and A. Ritz, "Proving a secluded $U(1)$ at $B$ factories", Phys. Rev. D 79, 115008 (2009).

[10] I. Jaegle et al. [Belle Collaboration], "Search for the dark photon and the dark Higgs boson at Belle", Phys. Rev. Lett. 114, 211801 (2015).

[11] J. P. Lees et al. [BaBar Collaboration], "Search for Low-Mass Dark-Sector Higgs Bosons", Phys. Rev. Lett. 108, 211801 (2012).

[12] G. J. Feldman and R. D. Cousins, Phys. Rev. D 57, 3873 (1998).

[13] D. Babusci et al. (KLOE-2 Collaboration), Phys. Lett. B 720, 111 (2013).

[14] R. Essig, J. Mardon, M. Papucci, T. Volansky, and Y. Zhong, "Constraining light dark matter with low-energy $e^{+} e^{-}$colliders", J. High Energy Phys. 11, 167 (2013). 